\title{
Harmonic and Reactive Power Compensation as Ancillary Services in Inverter Based Distributed Generation
}

\author{
Milan Prodanović $^{*}$, Karel De Brabandere ${ }^{* *}$, Jeroen Van den Keybus **, \\ Tim Green *, Johan Driesen ** \\ * Dept of EEE, Imperial College London, \\ ** Dept. of EE, Catholic University of Leuven
}

Abstract - The introduction of distributed generation, DG, into low voltage networks opens up the possibility of supplying ancillary services to aid network management and the maintenance of power quality. DG itself can cause voltage magnitude difficulties when injecting real power into networks with high $R / X$ ratios but control of reactive power injection can help overcome this. Continuous control and support only at voltage limit conditions (to avoid unnecessary reactive power flows in normal conditions) are explored. Relatively high-impedance low-voltage networks are prone to harmonic distortion from non-linear loads. A variety of control methods that emphasise either harmonic line flows or local voltage distortion are examined and a compromise method based on resistance emulation is shown to be effective. Experimental results from a single-phase laboratory network and 2kVA inverter are used to illustrate how these additional control functions can be integrated into the existing control scheme for real power management. Decomposition of observed voltages and currents into harmonic terms that are phasesynchronised to the grid voltage is a challenge in real-time systems. Kalman observers are used to achieve this with the additional advantage of avoiding explicit phase-locking while producing quadrature components useful in instantaneous calculation of reactive power and in providing feed-forward compensation terms.

Index terms: power quality, inverter, micro-grid, distributed generation 


\section{Introduction}

Small-scale distributed generation (DG) often requires an inverter interface to the local distribution network. The power ratings of these inverters are too small for them to have any impact on system frequency but their power rating can be relatively large compared to the short-circuit apparent power of a low-voltage grid and so they can contribute to local voltage control both in terms of magnitude and waveform quality. Further, if local inverters supply the bulk of the local real power demand without supplying reactive and harmonic current then an unreasonable burden is placed on the network operator. With that in mind, it is useful to examine how DG inverters could contribute ancillary services such as reactive power and harmonic compensation.

There are two main approaches to control of a voltage source inverter in grid connection. The first approach is based on mimicking of the operation of the synchronous generator and is explained in [1], [2]. This provides a simple means of achieving active and reactive power control in steady-state and ensures compatibility with existing network operations. However, a number of difficulties or missed opportunities result. Because only the voltage is controlled, there is no direct control over the output currents of the inverter. Voltage harmonics present in the grid can drive large uncontrolled currents through the low output impedance of a voltage source inverter. Further, transient disturbances in the network can cause large current excursions. The short-time overload capacity of an inverter is small compared to a synchronous generator of the same rating so these transient currents are potentially damaging. Either the inverter ratings must be increased to allow for the extra current or control measures must be added to avoid them. An example of a missed opportunity is the creation of a sinusoidal set of internal inverter voltages. With appropriate control, the inverter could provide some compensation in the harmonic frequency range to improve the quality of the voltage in the local grid. The resistor-emulation form of harmonic compensation is a method that can be incorporated into a voltage controlled inverter $[3],[4],[5],[6]$. The alternative approach to inverter operation sees it operated as a current source [7], [8]. By generating closely controlled and harmonic-free currents, this method shows advantages in terms of the current quality, stability, full utilisation of ratings and protection. However, current source operation does not make a contribution to improving the harmonic quality of the local voltage.

In this paper, a new inverter control method for harmonic compensation will be presented and compared with the two existing methods. These three methods span the range from the current quality improvement to the voltage quality improvement. Ultimately, it is left to the distribution network operator to assess the balance between local voltage quality and avoidance of harmonic line currents 
and to assess the value of having harmonic suppression provided by DG rather than network equipment. By making available a choice of control methods, harmonic compensation can be a flexible and viable ancillary service.

Reactive power compensation is available under most forms of power regulation used with inverters. Reactive power control is a particularly important ancillary service in low-voltage grids. The voltage magnitude can change significantly when active power is exported into a network with a high resistance to reactance ratio. To some extent this can be compensated by export or import of reactive power using any remaining available inverter rating. This paper will present and discuss three different modes of reactive power compensation which from a network operators point of view give choices over the extent to which and the circumstances in which voltage control is taken on by DG.

\section{Harmonic Control Strategies}

The form of DG connection being considered is shown in Fig. 1. An inverter supplies a set of local loads and has a connection to the local distribution network. The inverter system consists of an inverter bridge and an $L C$ filter. The network connection is assumed to be short and of relatively low impedance (represented by $Z_{\text {line }}$ ). The main grid is represented by a harmonic-free voltage source and a relatively large impedance $Z_{G}$ (as it would be in a remote connection). In the general case, the local load consists of a combination of linear and non-linear loads that produce harmonically distorted currents and consume reactive as well as active power.

Inverters are normally designed to withstand a maximum current of around $200 \%$ to $300 \%$ of the nominal RMS value. This excess current capacity is required to supply in-rush current to some forms of load and to provide some degree of fault current to activate protection systems. For the vast majority of its life, the inverter will operate with current well below this value and the extra capacity provides an opportunity for the inverters to generate harmonic current on top of the fundamental frequency component and take part in the harmonic compensation.

Only available harmonic directives for DG interconnection to the grid are given in [9], [10]. They are not restrictive regarding the harmonic generation and can be taken only as a rough guideline and many distribution companies already imposed much stricter standards of supply.

\subsection{Inverter Output Current Control}

The aim of this control strategy is to generate inverter output current, $i_{O}$, that is harmonic-free under all circumstances including, in particular, the case of the voltage at the point of the connection, $v_{O}$ 
being harmonically distorted, [7], [8]. Under such control, the inverter presents an infinite impedance to grid voltage harmonics and, provided the control is accurate, the inverter current will be harmonic free. The inverter contributes power according to the product of its own (sinusoidal) current and the fundamental component of the local grid voltage. Any harmonic power (active or non-active) required by the load must be sourced by the flow of harmonic current from the grid. The voltage at the point of connection will be harmonically distorted to an extent dependent on the grid impedance and the magnitude of the harmonic load current. The controller makes no attempt to compensate this distortion.

In order for the inverter output current to be harmonic-free, the sum of the harmonic current driven through the output capacitor (by the harmonic voltage at the point of connection) must be compensated by a harmonic current contribution through the filter inductor. Equations (1) and (2) show how this compensating current is calculated.

$$
\begin{gathered}
\underline{I_{O}}\left(n \omega_{O}\right)=\underline{I_{L}}\left(n \omega_{O}\right)-j n \omega_{O} C \underline{V_{O}}\left(n \omega_{O}\right)=0 \\
\underline{I_{L}}\left(n \omega_{O}\right)=j n \omega_{O} C \underline{V_{O}}\left(n \omega_{O}\right)
\end{gathered}
$$

\subsection{Resistor Emulation}

An inverter can be operated to emulate a resistance at harmonic frequencies by drawing a current proportional to the harmonic voltage. Several reports exist [3], [4], [5], [6] of means to choose a resistor value to improve the overall voltage quality (normally expressed as the THD). Resistor emulation may be viewed as providing a sink for the harmonic currents originating in non-linear loads and, because this reduces the magnitude of harmonic current in the grid impedance, the local voltage quality improves. The inverter will have a non-sinusoidal output current (unless the voltage at the point of connection is perfectly sinusoidal). The output power of the inverter will contain terms due to the fundamental voltages and currents and also some power consumption (damping) at harmonic frequencies.

Equation (3) shows the relation between the desired value of the output current $i_{O}$, the voltage at the point of connection $v_{O}$ and the emulated harmonic resistance $R_{e m}\left(n \omega_{O}\right)$ and (4) shows the required value of filter inductor current $i_{L}$.

$$
\underline{I_{O}}\left(n \omega_{O}\right)=\underline{I_{L}}\left(n \omega_{O}\right)-j n \omega_{O} C \underline{V_{O}}\left(n \omega_{O}\right)=-\frac{V_{O}\left(n \omega_{O}\right)}{R_{e m}\left(n \omega_{O}\right)}
$$




$$
\underline{I_{L}}\left(n \omega_{O}\right)=j n \omega_{O} C \underline{V_{O}}\left(n \omega_{O}\right)-\frac{V_{O}}{R_{e m}\left(n \omega_{O}\right)}
$$

\subsection{Active Inverter Output Voltage Control}

Inverters are sometime operated as simple voltage sources by setting the bridge voltage $V_{I}$ to be sinusoidal. However, harmonic current drawn from the inverter will cause harmonic distortion of the voltage because of the output impedance of the bridge and filter. Here an active means of control of the filter output voltage is proposed to ensure it remains sinusoidal even in the presence of non-linear loads. The inverter (including the filter) presents a relatively low impedance to harmonic currents in comparison to the grid and distribution network. A dominant fraction of the harmonic current of the local load can be expected to be compensated by the inverter. Active voltage control can improve the quality of the local voltage when there is a significant impedance between the local loads and the main grid $Z_{G}$. If the inverter output voltage is maintained sinusoidal then only the fundamental component of inverter current carries real power.

For the output voltage to be harmonic-free, there should be no harmonic current flow in the filter capacitor and therefore the filter inductor current must provide all of the harmonic current required at the output, as expressed in (5).

$$
j n \omega_{O} C \underline{V_{O}}\left(n \omega_{O}\right)=0=\underline{I_{C}}\left(n \omega_{O}\right)=\underline{I_{L}}\left(n \omega_{O}\right)-\underline{I_{O}}\left(n \omega_{O}\right)
$$

There is potential for the filter capacitance to resonate with the line impedance and cause lightly damped oscillations. To combat this a resistive term can be added to the filter inductor current to provide damping, (6).

$$
\underline{I_{L}}\left(n \omega_{O}\right)=\underline{I_{O}}\left(n \omega_{O}\right)+j n \omega_{O} C \underline{V_{O}}\left(n \omega_{O}\right)-\frac{\underline{V_{O}}\left(n \omega_{O}\right)}{R_{e m u}\left(n \omega_{O}\right)}
$$

\section{Voltage Magnitude Control and Reactive Power Compensation Techniques}

When a distributed generator starts exporting power to a low-voltage grid, the voltage magnitude can change significantly because of the resistive portion of line impedance. In low voltage networks the $R / X$ ratio is normally large and significant over-voltage can occur near the point of DG connection. The inverter based generation can use its reactive power capacity to control the voltage at the point of connection. However, the present regulations generally prohibit direct voltage control by indepen- 
dent generation because of a lack of coordination with the network operators control. However, in future network operators may allow, or even require, reactive power generation or voltage magnitude control in order to form part of the overall network management. Active power control should not be compromised by the use of reactive power and the apparent power limit of the inverter must be respected.

Three scenarios for the reactive power compensation will be discussed. Some of methods of reactive power deployment require coordination from the network operator (by using a low-bandwidth communication link) whereas others can use preset references. The network operator may implement some form of active management control of the local distributed generation.

\subsection{Reactive Power Dispatch}

Where the operator has a reasonably extensive network monitoring system and can call upon several DG with reactive power control, the network operator may operate some form of reactive power dispatch by setting demands for the reactive power export. The goal of the inverter controller is then to follow the reactive power reference irrespective of the voltage level at the point of connection. The drawback of this method is that the local voltage is not controlled in real-time. It is the task of the network operator to maintain the local voltage levels in slow acting control. It is important to emphasise that this operation is completely independent of the voltage control operation at the higher voltage (such as MV tap changers) and its aim is to compensate the reactive power locally (within a local LV network) and it that way support the higher level voltage control.

\subsection{Voltage Magnitude Control}

If there is sufficient impedance between the inverter and the grid connection (and the network operator allows it) then the inverter can control the voltage magnitude at the point of connection through manipulation of reactive power. The voltage level can be preset or actively changed by the operator. If the limit of the inverter apparent power is reached then control of the voltage will not be possible. The advantage of this method is that the local voltage magnitude is maintained despite the disturbance to the line voltage drops caused by changes in the active power export through resistive lines. Operator intervention may be required to adjust the reference voltage if excessive reactive power generation is noted. 


\subsection{Dead-band Voltage Control}

When the network operator is not able to provide controller references for reactive power or voltage, the inverter can be set to intervene only if the voltage magnitude exceeds (or perhaps approaches) the tolerance limits of the network. For most of the time the voltage will be in a dead-band where no control action is applied. This ensures that the inverter does not attempt to generate large magnitudes of reactive power, possibly unhelpfully, in normal operation and only intervenes in abnormal conditions. Once the voltage magnitude reaches either upper or lower limit, the reactive power generation will be activated to generate either capacitive or inductive reactive power to prevent the voltage magnitude exceeding the limit and in this way support the voltage control. If the voltage is such that the apparent power limit of the inverter is reached then the inverter will continue to support the voltage control in its attempt to restore nominal conditions by exporting the maximum reactive power. The advantage of this method is that the distributed resource does not require any intervention from the network control centre or provision of a communication link. The unit can operate autonomously within the preset voltage limits at the expense of no voltage magnitude control within these limits.

\section{Controller Design}

It is a basic requirement that an inverter maintains synchronism with the network voltage and it will always be necessary that some control is exercised over the export of real power. (In this work it was assumed that the capacity of the DG is small compared to that of the main grid and that the DG would not participate in frequency control.) To the two basic functions we wish to add ancillary services of reactive power (or voltage magnitude) control and harmonic compensation.

Fig. 2 shows the overall control block diagram to be used in this design. The approach adopted is that the voltage source inverter will be subject to fast acting current control and that all of the overall control objectives can be satisfied by applying appropriate current references (summed to give $i_{L}^{*}$ ) to this current controller. Observers (based on Kalman filters) are used to decompose the observed voltages and currents at the output of the inverter. The observers are set to create in-phase and quadrature observations. Fundamental components are used in the real and reactive power control (producing reference component $i_{R}$ ) and harmonic components are used for harmonic compensation (producing reference harmonic components $i_{K}^{3, ., n}$ ). 


\subsection{Power and Voltage Control}

In principle, real power export from the inverter can be achieved in open loop: the power reference would be divided by the prevailing network voltage to create the current reference to be passed to the current control loop. The impact of un-modelled losses and current regulator inaccuracies can be reduced by measuring the actual output power and using a PI controller to regulate the power. Fig. 3 shows the structure of the real and reactive power controllers. The several options for reactive power deployment are shown with a switch for mode selection.

The real power export is considered to be only the real power arising from fundamental frequency current and is calculated from (7).

$$
P(t)=\frac{1}{2}\left(v_{O}^{1}(t) i_{O}^{1}(t)+v_{O j}^{1}(t) i_{O j}^{1}(t)\right)
$$

Measurements of reactive power (following [11]) and voltage magnitude also concern only fundamental frequency components, (8) and (9).

$$
\begin{gathered}
Q(t)=\frac{1}{2}\left(v_{O}^{1}(t) i_{O j}^{1}(t)-v_{O j}^{1}(t) i_{O}^{1}(t)\right) \\
V_{O}^{1}(t)=\sqrt{\left(v_{O}^{1}(t)\right)^{2}+\left(v_{O j}^{1}(t)\right)^{2}}
\end{gathered}
$$

The three different modes of reactive power compensation are those introduced in Section 3. With the switch in position $a$ ), the reactive power export is regulated at the desired level of $Q^{*}$. With the switch in position $b$ ), voltage magnitude is continuously regulated by using voltage error as an input to a PI controller that sets the reactive power demand. Option $c$ ) is similar to $b$ ) except that a large dead-band exists in normal operation and reactive power is only used if the voltage reaches its limit values.

$$
\hat{Q}= \begin{cases}Q_{\max } \frac{V_{\text {Oliml }}-V_{O}^{1}}{V_{\text {diff }}} & V_{O}^{1}<V_{\text {Oliml }} \\ 0 & V_{\text {Oliml }} \leq V_{O}^{1} \leq V_{\text {Olimu }} \\ -Q_{\max } \frac{V_{O}^{1}-V_{\text {Olimu }}}{V_{\text {diff }}} & V_{\text {Olimu }}<V_{O}^{1}\end{cases}
$$

The real and reactive power demands can be translated to a demand for inverter current, $i_{R}$ by dividing the complex power by the voltage vector. The voltage observer is designed to yield the voltage together with a quadrature component so that angle information is available and explicit phase locking is not required. A term is added to the current reference to account for the current expected to flow through the filter capacitor as expressed in (11) and (12). 


$$
\begin{aligned}
& i_{R}^{1}(t)=2 \frac{v_{O}^{1}(t) \hat{P}-v_{O j}^{1}(t) \hat{Q}}{\left(V_{O}^{1}\right)^{2}}+\omega_{O} C v_{O j}^{1}(t) \\
& i_{R j}^{1}(t)=2 \frac{v_{O j}^{1}(t) \hat{P}+v_{O}^{1}(t) \hat{Q}}{\left(V_{O}^{1}\right)^{2}}-\omega_{O} C v_{O}^{1}(t)
\end{aligned}
$$

\subsection{Current Controller}

The current in the filter inductor is controlled with a high-gain proportional controller which uses feedforward to overcome disturbances introduced by the filter capacitor (output) voltage. The controller output is applied to the inverter via a standard PWM system.

$$
v_{I}^{*}(t)=v_{O e}(t)+K\left(i_{L}^{*}(t)-i_{L}(t)\right)
$$

The inductor current, $i_{L}$ at each harmonic frequency can be readily calculated from the voltage difference across the inductor, (14).

$$
\underline{I_{L}}\left(n \omega_{O}\right)=\frac{V_{I}\left(n \omega_{O}\right)-\underline{V_{O}}\left(n \omega_{O}\right)}{j n \omega_{O} L+R_{L}}
$$

Assuming that the feed-forward term is accurate, the current control loop achieves the steady-state transfer ratio $H_{C}$ given in (15).

$$
\underline{H_{C}}\left(n \omega_{O}\right)=\frac{I_{L}^{*}\left(n \omega_{O}\right)}{\underline{I_{L}}\left(n \omega_{O}\right)}=\frac{1}{1+R_{L} / K+j n \omega_{O} L / K}
$$

To cancel the effect of this steady-state control error, the inverse of this transfer ratio can be used to pre-compensate (scale) the fundamental and harmonic components of the current reference as shown in (16) and Fig. 2. Finally, all the harmonic components of the current reference are summed to form $i_{L}^{*}$

$$
i_{L}^{* n}(t)=\left.i_{K}^{n}(t) \frac{1}{H_{C}\left(n \omega_{O}\right)}\right|_{r e a l}+\left.i_{K j}^{n}(t) \frac{1}{H_{C}\left(n \omega_{O}\right)}\right|_{i m a g}
$$

\subsection{Voltage and Current Observation}

Essential elements of the proposed control scheme are the decomposition of the voltage and current waveforms into fundamental and harmonic terms; the identification of real and reactive (fundamental) power and creation of current references with active and reactive components. It is common to apply the DFT to decompose harmonics and, if phase locked, this can also separate in-phase and quadrature 
components (although other approaches are also reported [12]). Such methods are computationally complex and real time implementation is demanding.

An alternative method proposed in [13] employs a Kalman observer to identify the harmonic components. Several existing applications have been reported including application of the method to active power filters and parallel connected inverters [4], [8], [14]. The algorithm is a real-time pointby-point (not a block) process of digital filtering in which the updates of the observed states are available in real-time at each sample interval. The observers can be designed to converge with acceptable accuracy within a few cycles of the fundamental frequency. In this application, the inputs to the Kalman observers are the output voltage and current $v_{O}$ and $i_{O}$. The observers produce the harmonic components and corresponding orthogonal (90 phase-leading) signals as is shown in (17) and (18).

$$
\begin{gathered}
v_{O}^{n}(t)=V_{O}^{n} \cos \left(n \omega_{O} t+\phi_{V}^{n}\right) \\
i_{O}^{n}(t)=I_{O}^{n} \cos \left(n \omega_{O} t+\phi_{I}^{n}\right) \\
v_{O j}^{n}(t)=-V_{O}^{n} \sin \left(n \omega_{O} t+\phi_{V}^{n}\right) \\
i_{O j}^{n}(t)=-I_{O}^{n} \sin \left(n \omega_{O} t+\phi_{I}^{n}\right)
\end{gathered}
$$

This method of harmonic measurement is sensitive to changes in the grid frequency. If the frequency changes from its nominal value, the Kalman observers produce sinusoidal output at the new frequency but with some error in the the magnitudes and phases. The Kalman observer can be set in such a way as to produce a small error over the expected frequency range (perhaps $+/-1 \%$ of the nominal frequency as in [4]). If an extended range or more accurate calculation is required, it is possible to either synchronise the sampling with the grid frequency or use a phase-locked loop to update the calculation matrices.

\subsection{Harmonic Compensation}

Three forms of harmonic compensation were defined in Section 2: current quality control, resistor emulation and output voltage quality control. Each method can be used to create a compensation current component, $i_{K}$ to add to the current reference for the power regulation, $i_{R}$. Equations (19), (20), (21) express the three forms of reference required. In each case in-phase and quadrature components are calculated for each harmonic. 


$$
\begin{gathered}
i_{K}^{n}(t)=n \omega_{O} C v_{O j}^{n}(t) \\
i_{K j}^{n}(t)=-n \omega_{O} C v_{O}^{n}(t) \\
i_{K}^{n}(t)=n \omega_{O} C v_{O j}^{n}(t)-\frac{1}{R_{e m u}} v_{O}^{n}(t) \\
i_{K j}^{n}(t)=-n \omega_{O} C v_{O}^{n}(t)-\frac{1}{R_{e m u}} v_{O j}^{n}(t) \\
i_{K}^{n}(t)=n \omega_{O} C v_{O j}^{n}(t)-\frac{1}{R_{e m u}} v_{O}^{n}(t)+i_{O}^{n}(t) \\
i_{K j}^{n}(t)=-n \omega_{O} C v_{O}^{n}(t)-\frac{1}{R_{e m u}} v_{O j}^{n}(t)+i_{O j}^{n}(t)
\end{gathered}
$$

Because calculation of the capacitive element of the compensation reference employs the assumed grid frequency, the compensation may not be entirely accurate, especially at higher harmonics but only a small degradation of the total harmonic quality of the voltages or currents is to be expected.

The calculation of the compensation reference can be implemented efficiently as a scalar product between the orthogonal components of voltage or current (stored in a vector format) and a vector of pre-calculated cancellation constants. The harmonic compensation block in Fig. 2 shows multiplcation of the input vector of voltage or current harmonic components by the vectors of constants $C_{v_{O}}, C_{v_{O j}}$, $C_{i_{O}}$ and $C_{i_{O j}}$. To switch between the different modes of harmonic compensation, it is only required to change the vector of constants.

\section{Experimental Results}

A rapid prototyping system [15] was used to implement the various control algorithms on a standard single-phase inverter. The parameters of the experimental setup are shown in Table 1. The inverter was connected to the grid available in the laboratory and to a range of non-linear loads connected such as PCs and light-ballasts. The Kalman observers were set to extract fundamental, $3^{\text {rd }}, 5^{\text {th }}$ and $7^{\text {th }}$ harmonic components and, therefore, only those components were actively compensated during the experiments. 


\begin{tabular}{|l|c|}
\hline \multicolumn{1}{|c|}{ Parameter } & Value \\
\hline \hline Nominal Grid Voltage & $230 \mathrm{~V}_{R M S}$ \\
\hline Nominal Voltage Magnitude & $325 \mathrm{~V}$ \\
\hline Inverter Power Rating & $2 \mathrm{kVA}$ \\
\hline Filter Inductance $L$ & $4.1 \mathrm{mH}$ \\
\hline Series Resistance of The Filter Inductor $R_{L}$ & $0.95 \Omega$ \\
\hline Filter Capacitance $C$ & $10 \mu \mathrm{F}$ \\
\hline Current Feedback Gain & $14.6 \mathrm{~V} / A$ \\
\hline Emulated Resistance $R_{e m u}$ & $0.33 \Omega$ \\
\hline
\end{tabular}

Table 1: Parameters of The Experimental Setup

\subsection{Harmonic Compensation}

Fig. 4 and 5 show the inverter output voltage $v_{O}$ and the inverter current $i_{O}$ for different modes of harmonic compensation. The test was conducted under an active power demand of $1.5 \mathrm{~kW}$ and zero reactive power demand.

Harmonic analysis was conducted to quantify the performance of the three methods and the results are shown in Table 2. As expected, the active inverter output voltage control shows the lowest distortion in the voltage but a high harmonic current contribution from the inverter. The $3^{\text {rd }}, 5^{\text {th }}$ and $7^{t h}$ voltage harmonic components are significantly improved and an almost sinusoidal waveform was obtained. In order to improve the quality of the local voltage, it was necessary to produce highly distorted currents to compensate for the local harmonic loads as explained in Section 2.3. Significant $5^{\text {th }}$ harmonic current flows from the output of the inverter in order to maintain the voltage sinusoidal despite significant $5^{\text {th }}$ harmonic voltage component in the laboratory grid voltage (as observed in the no-compensation case).

The inverter output current control did not affect the voltage quality but did provide very low distortion inverter current. The results for the resistance emulation method showed improved voltage harmonic performance.

\subsection{Reactive Power Control}

The voltage magnitude control was tested by applying a step-change of $1 \mathrm{~kW}$ to the active power demand. Because the laboratory network has a relatively high $\mathrm{R} / \mathrm{X}$ ratio, this caused a significant disturbance of the voltage magnitude (2.5V compared to $325 \mathrm{~V}$ of nominal magnitude) which the 


\begin{tabular}{|c|c||c|c|c|c||c|}
\hline \multirow{2}{*}{$\begin{array}{c}\text { Control } \\
\text { Method }\end{array}$} & & \multirow{2}{*}{$\begin{array}{c}\text { RMS } \\
{[\mathbf{V}][\mathbf{A}]}\end{array}$} & \multicolumn{3}{|c||}{ Harmonic [\%] } & Total \\
\cline { 4 - 7 } & & 3 & 5 & 7 & THD [\%] \\
\hline \hline \multirow{2}{*}{$\begin{array}{c}\text { No } \\
\text { Comp. }\end{array}$} & $v_{O}$ & 235.7 & 0.22 & 2.28 & 1.14 & 2.67 \\
\cline { 2 - 7 } & $i_{O}$ & 6.42 & 1.50 & 1.70 & 1.00 & 2.02 \\
\hline \hline \multirow{2}{*}{$\begin{array}{c}\text { Current } \\
\text { Quality }\end{array}$} & $v_{O}$ & 236.3 & 0.24 & 2.31 & 1.05 & 2.62 \\
\cline { 2 - 7 } & $i_{O}$ & 6.04 & 0.71 & 0.90 & 0.08 & 1.44 \\
\hline \hline \multirow{2}{*}{$\begin{array}{c}\text { Resistor } \\
\text { Emulation }\end{array}$} & $v_{O}$ & 235.3 & 0.05 & 0.43 & 0.19 & 0.68 \\
\cline { 2 - 7 } & $i_{O}$ & 6.81 & 5.12 & 30.88 & 11.00 & 31.89 \\
\hline \hline \multirow{2}{*}{$\begin{array}{c}\text { Voltage } \\
\text { Quality }\end{array}$} & $v_{O}$ & 235.1 & 0.02 & 0.15 & 0.09 & 0.48 \\
\cline { 2 - 7 } & $i_{O}$ & 6.87 & 5.83 & 33.80 & 11.38 & 36.26 \\
\hline
\end{tabular}

Table 2: Harmonic Analysis of Three Methods For Harmonic Compensation

voltage control loop seeks to correct by injecting reactive power. Fig. 6 shows that at each step the voltage is disturbed but is brought back to the set point as the reactive power is adjusted. The response has been designed to be slightly under damped but takes less than $1 s$ to settle.

The dead-band method of voltage control was tested by varying the local grid voltage magnitude with a VARIAC. The limits of the dead-band were $325 \pm 2.5 \%$, i.e. $318 \mathrm{~V}$ and $333 \mathrm{~V}$ and the inverter was set to export $1 \mathrm{~kW}$ of real power. Fig. 7 shows the experimental results. Initially, the grid voltage magnitude was within the limits and decreased slowly. No reactive power is exchanged until the lower voltage limit is reached. On reaching the limit, the reactive power export increased to prevent further voltage decrease. There comes a point at which the maximum apparent power capability of the inverter is reached and the reactive power saturates. It is then no longer possible to maintain the voltage at its lower limit. On increasing the local grid voltage, similar behaviour is observed at the upper limit. The test confirms that this method sucessfully provided support for the voltage control at the voltage limits within its reactive power capacity. Also, no reactive power is exchanged with the grid provided the network conditions are such that the voltage would be within the limits anyway.

\section{Conclusion}

Low voltage networks with relatively high and resistive network impedances are prone to harmonic distortion if non-linear loads are present and prone to voltage magnitude errors if real power flows are reversed by DG. However, inverter-interfaced DG can provide means to suppress both problems. 
This paper has demonstrated through analysis and experiment that control functions can be added to DG inverters to provide forms of reactive power compensation and harmonic suppression as ancillary services.

In tackling harmonic distortion there is a need to decide whether the voltage quality provided to consumers or the reduction of harmonics in line flows is the most important consideration. To some extent these are competing objectives. For instance, maintaining a low distortion local voltage in a case where the grid voltage is distorted will cause significant harmonic current flow in the connecting line. Three means of tackling harmonic distortion have been examined one of which, resistance emulation, provides a compromise solution that improves local voltage quality without causing large harmonic current flows.

Three different strategies for reactive power compensation have been presented. Depending on the required operating mode, it has been discussed how to use the reactive power capacity of an inverter interfaced DG to support the control of the voltage magnitude in LV networks.

Both reactive power compensation and harmonic suppression require a decomposition of voltages and currents into harmonic terms expressed as vectors phase-synchronised to the grid voltage. It has been demonstrated that a Kalman observer can perform the decomposition and produce in-phase and quadrature components of single-phase systems without explicit phase-locking. Further, the method uses real-time point-by-point rather than off-line block processing (DFT) and has a realistic computational burden for real-time implementation where a limited number of harmonics are of concern. 


\section{References}

[1] P. Kundur, Power System Stability and Control. McGraw-Hill, Inc., 1994.

[2] M. Chandorkar, D. Divan, and R. Adapa, "Control of Parallel Connected Inverters in Stand-Alone AC Supply Systems," IEEE Tran. on Industry Applications, vol. 29, no. 1, pp. 136-143, 1993.

[3] H. Akagi, "Control Strategy and Site Selection of A Shunt Active filter for Damping of Harmonic Propagation in Power Distribution Systems," IEEE Trans. on Power Delivery,, vol. 12, pp. 354-363, January 1997.

[4] K. De Brabandere, B. Bolsens, J. Van den Keybus, J. Driesen, and R. Belmans, "A Voltage and Frequency Droop Control Method for Parallel Inverters," 35th Annual IEEE Power Electronics Specialists Conference, pp. 2501-2507, June 2004 .

[5] N. Pogaku and T. C. Green, "Harmonic Mitigation Throughout a Distribution System: A Distributed-Generator System Based Solution," Accepted for publication in IEE Proceeding GTD, 2006.

[6] N. Pogaku and T. C. Green, "Application of Inverter-Based Distributed Generators for Harmonic Damping Throughout a Distrubution Network," 36th Annual IEEE Power Electronics Specialists Conference, pp. 1922-1927, June 2005.

[7] M. Prodanović and T. Green, "Control and Filter Design Of Three-Phase Inverters For High Power Quality Grid Connection," IEEE Transactions on Power Electronics, vol. 18, pp. 373-380, Jan 2003.

[8] B. Bolsens, K. De Brabandere, J. Van den Keybus, J. Driesen, and R. Belmans, "Model-Based Generation of Low Distortion Currents in Grid-Coupled PWM-Inverters Using an LCL Output Filter," 35th Annual IEEE Power Electronics Specialists Conference, pp. 4616-4622, June 2004.

[9] CENELEC, Voltage Characteristics of Electricity Supplied by Public Distribution Systems. BSI, 2000.

[10] IEEE SCC21, IEEE P1547 Std Draft 06 Standard for Distributed Resources Interconnected with Electric Power Systems. IEEE, 2000.

[11] H. Akagi, Y. Kanazawa, and A. Nabae, "Intantaneuos Reactive Power Compensators Comprising Switching Devices without Energy Storage Components," IEEE Tran. on Industry Applications, vol. IA - 20, pp. 625-630, May/Jun 1984.

[12] M. Karimi-Ghartemani, H. Mokhtari, M. R. Iravani, and M. Sedighy, "A Signal Processing System for Extraction of Harmonics and Reactive Current of Single-Phase Systems," IEEE Trans. on Power Delivery,, vol. 19, pp. 979-986, July 2004 .

[13] A. A. Girgis, W. B. Chang, and E. B. Makram, "A digital recursive measurement scheme for on-line tracking of power system harmonics," IEEE Trans. on Power Delivery,, vol. 6, pp. 1153-1160, June 1991.

[14] J. Barros and E. Pérez, "An Adaptive Method for Determining the Reference Compensating Current in Single-Phase Shunt Active Power Filters," IEEE Trans. on Power Delivery, vol. 18, pp. 1578-1580, October 2003.

[15] J. Van den Keybus, B. Bolsens, K. De Brabandere, J. Driesen, and R. Belmans, "DSP and FPGA Based Platform for Rapid Prototyping of Power Electronic Converters and Its Application to A Sampled-Data Three-Phase Dual-Band Hysteresis Current Controller," IEEE 33rd Annual Power Electronics Specialists Conference, vol. 4, pp. 1722-1727, June 2002. 


\section{List of Figures}

1 Inverter Connection To Local Loads and Utility Grid . . . . . . . . . . . . . . . . 17

2 A Schematic Diagram of the Control System for a DG Inverter Incorporating Ancillary

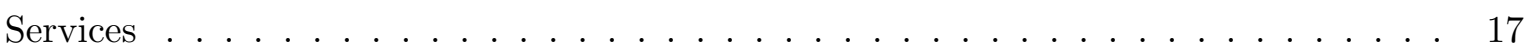

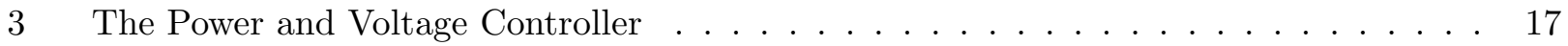

4 Inverter Output Voltage Waveforms $v_{O}$ For The Three Harmonic Compensation Methods 18

$5 \quad$ Inverter Output Current Waveforms $i_{O}$ For The Three Harmonic Compensation Methods 18

$6 \quad$ Response of reactive power controller to voltage disturbance casued by steps in real

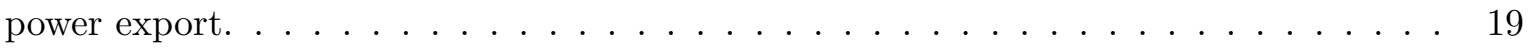

$7 \quad$ Response of Dead-band Voltage Controller to Variation of Local Grid Voltage . . . . . 19 


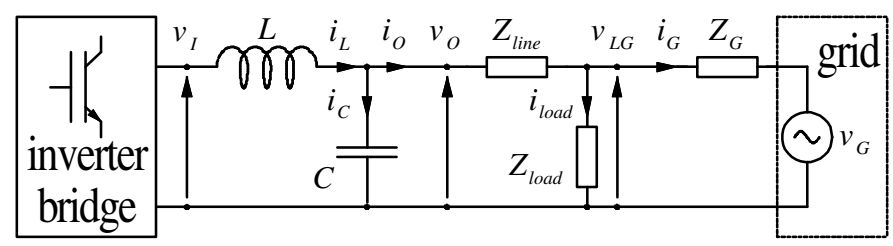

Figure 1: Inverter Connection To Local Loads and Utility Grid

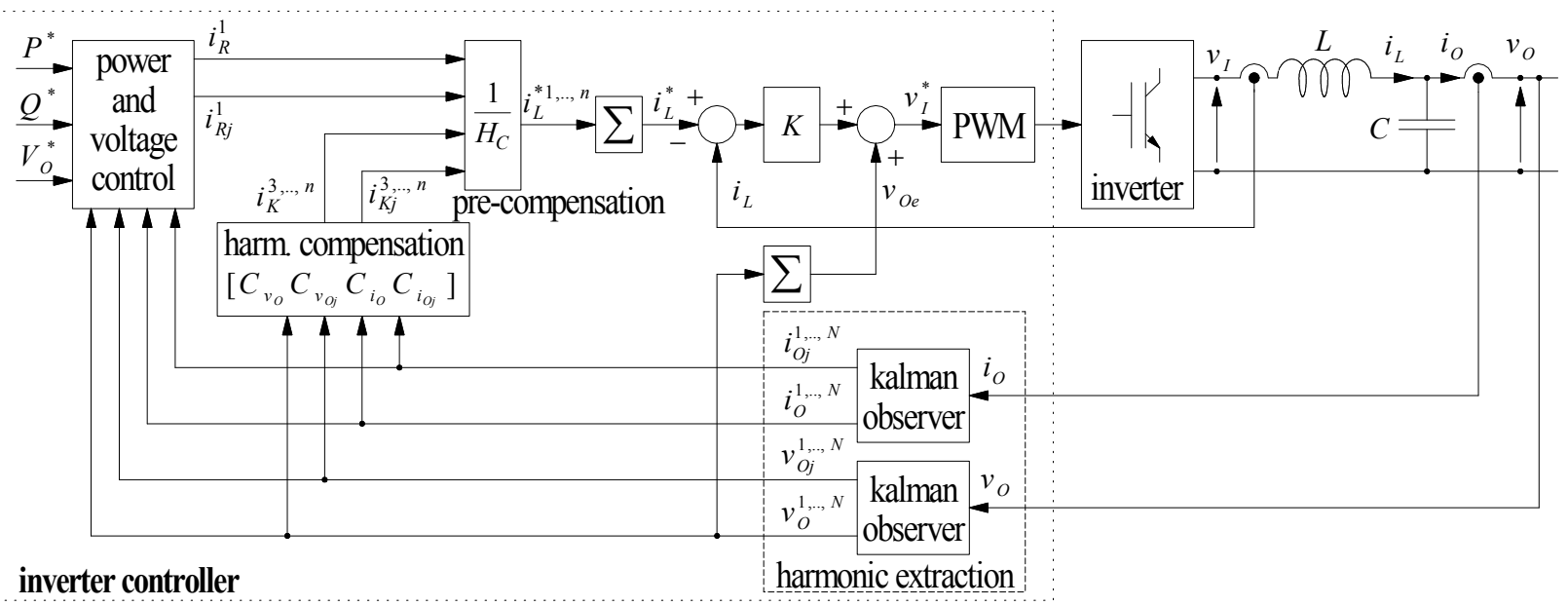

Figure 2: A Schematic Diagram of the Control System for a DG Inverter Incorporating Ancillary Services

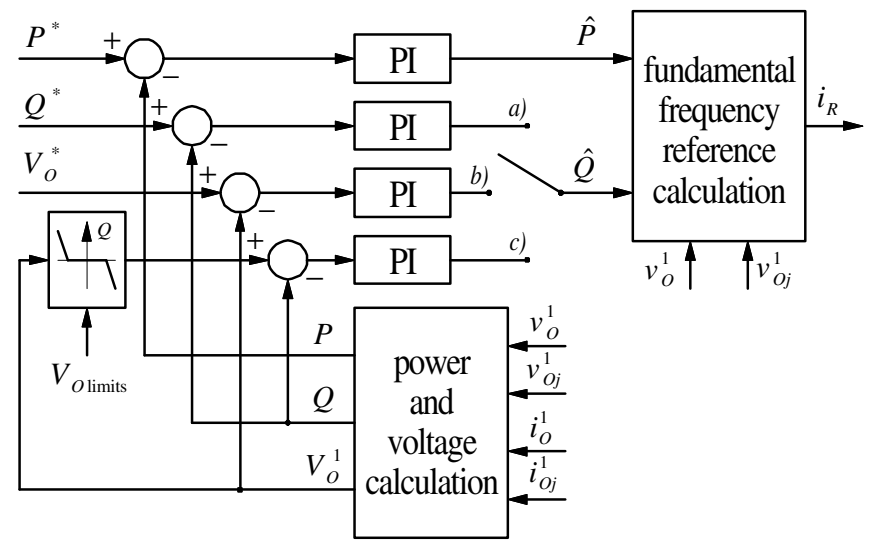

Figure 3: The Power and Voltage Controller 


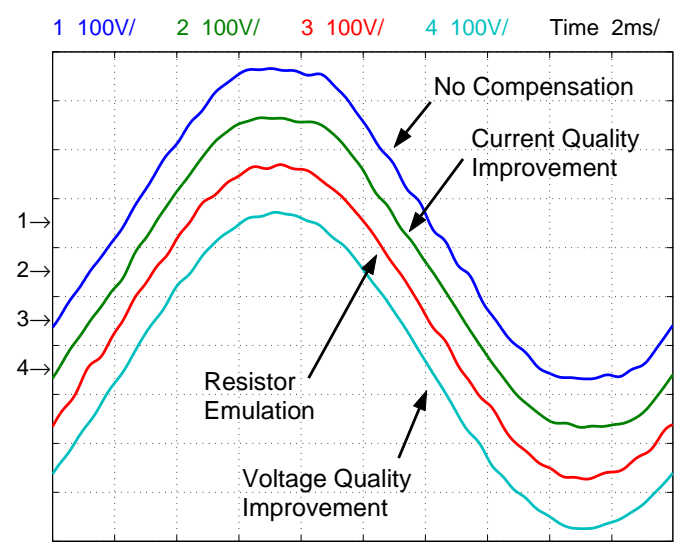

Figure 4: Inverter Output Voltage Waveforms $v_{O}$ For The Three Harmonic Compensation Methods

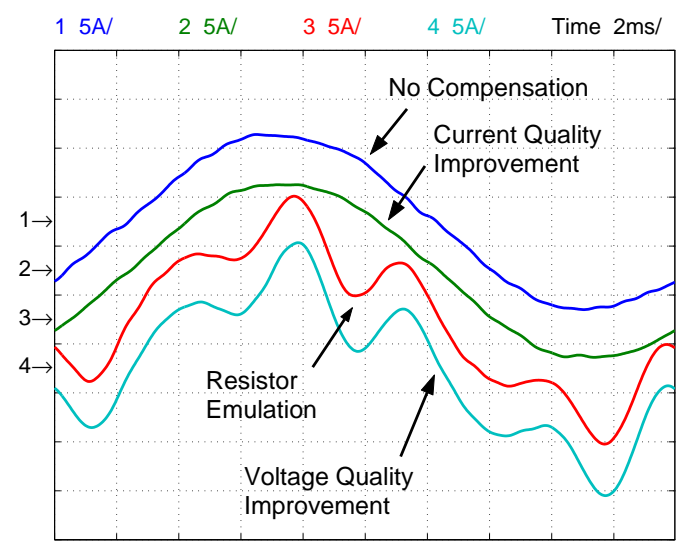

Figure 5: Inverter Output Current Waveforms $i_{O}$ For The Three Harmonic Compensation Methods 


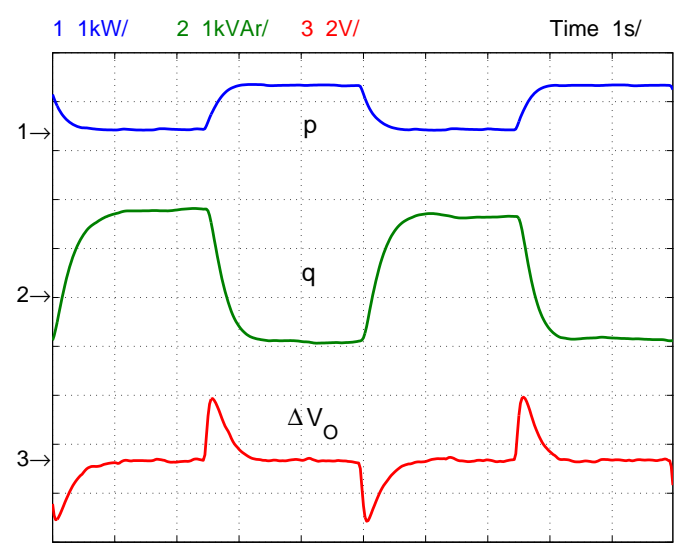

Figure 6: Response of reactive power controller to voltage disturbance casued by steps in real power export.

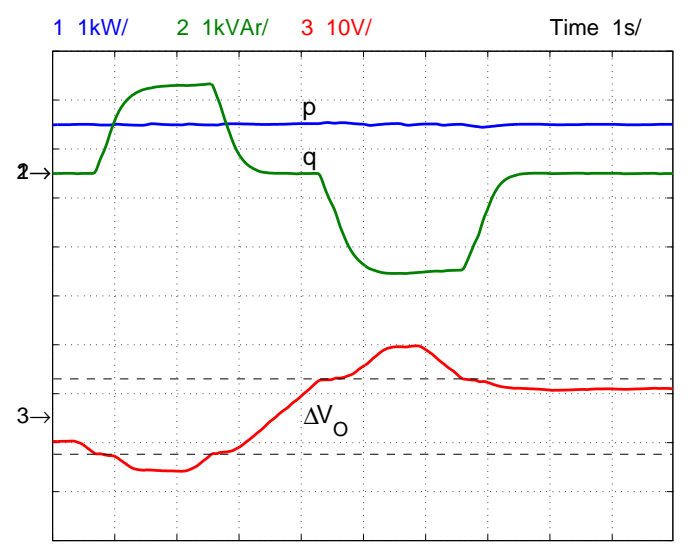

Figure 7: Response of Dead-band Voltage Controller to Variation of Local Grid Voltage 